\title{
Effect of Cooling Rate on the Structure of Sodium Borate Glasses
}

\author{
G. Ennas, A. Musinu, G. Paschina, G. Piccaluga, and G. Pinna \\ Dipartimento di Scienze Chimiche, Cagliari, Italy
}

Z. Naturforsch. 48a, 599-604 (1993); received January 19, 1993

\begin{abstract}
Sodium borate glasses with two different compositions have been prepared using cooling rates in the range $7 \cdot 10^{-3}{ }^{\circ} \mathrm{C} / \mathrm{s}-2 \cdot 10^{2}{ }^{\circ} \mathrm{C} / \mathrm{s}$. Structural characterization has been carried out by $\mathrm{X}$-ray diffraction, and glass transition temperatures have been measured by Differential Scanning Calorimetry. The samples exhibit slightly different density values and glass transition temperatures. However, radial distribution functions appear extremely similar and the sodium coordination shell does not change in going from fast to slowly quenched glasses, showing that the short and medium range order is not affected by thermal treatment.
\end{abstract}

Key words: Glass, structure, diffraction.

\section{Introduction}

Glasses, though kinetically stable systems, are outside thermodynamic equilibrium. Therefore their state is not univocally defined by thermodynamic variables but also depends on the preparation process. Though this is well known, there are very few studies correlating different preparation routes to structural differences and the letter ones to different properties.

Alkali borate glasses have been extensively studied because of their technological relevance as ionic conductors and because of their ease of vitrification under different thermal treatments, so that they appear suitable as model systems for structural investigations. In spite of the great amount of structural results, mainly related to the type of borate units and to their connections inside the network, less attention has been paid to the fact that the glasses investigated were often prepared in rather different ways [1-7], and therefore might have different physical properties. Only a few results about the effect of the thermal history on the structure of borate glasses are reported. For example, small but characteristic spectral differences have been observed in a Raman investigation [6] on the effect of the melt temperature on the structure of sodium borate glasses, which suggested the formation of new orthoborate units inside the network. Substantial structural differences are found in an X-ray diffraction study on $\mathrm{B}_{2} \mathrm{O}_{4}$ [4] obtained with different cooling rates, which indicates a more densely packed structure

Reprint requests to Dr. Anna Musinu, FAX: 003970669272 , Dipartimento di Scienze Chimiche, Via Ospedale 72, I-09 124 Cagliari/Italien. for the slowly quenched glass. This result has been supported by a low-frequency Raman study [3] on alkali borate glasses.

We recently investigated [8], by X-Ray Diffraction (XRD) and NMR measurements, the effects of quenching rate on the structure of $\mathrm{Na}_{2} \mathrm{O} \cdot n \mathrm{~B}_{2} \mathrm{O}_{3}$ $(n=2,3)$ glasses (named BONA2, and BONA3, respectively) and showed that the two different cooling rates $\left(5 \cdot 10^{-2}{ }^{\circ} \mathrm{C} / \mathrm{s}\right.$ and $\left.10^{\circ} \mathrm{C} / \mathrm{s}\right)$ used do not appreciably influence the short range order of these glasses. Small differences in the medium range order, however within the limits of the experimental uncertainty, needed further investigation. Moreover, as the explored range of cooling rates was in that case rather limited, we decided to repeat the experiments on glasses of the same composition but expanding the range from $7 \cdot 10^{-3}{ }^{\circ} \mathrm{C} / \mathrm{s}$ to $2 \cdot 10^{2}{ }^{\circ} \mathrm{C} / \mathrm{s}$. Glasses obtained by slow and very slow controlled quenching will be referred to as SQ and VSQ, and those obtained by very fast quenching will be referred to as VFQ.

Structural characterization of the glasses has been carried out by XRD and glass transition temperatures $\left(T_{\mathrm{g}}\right)$ have been measured by Differential Scanning Calorimetry (DSC).

\section{Experimental}

Reagent grade $\mathrm{Na}_{2} \mathrm{~B}_{4} \mathrm{O}_{7} \cdot 10 \mathrm{H}_{2} \mathrm{O}$ and $\mathrm{H}_{3} \mathrm{BO}_{3}$ were used as starting materials. Weighed amounts of the wanted mixtures were melted in a platinum crucible while the temperature was raised to $1200^{\circ} \mathrm{C}$ over a period of $130 \mathrm{~min}$ and kept at $1200^{\circ} \mathrm{C}$ for

0932-0784 / 93 / 0400-0599 \$01.30/0. - Please order a reprint rather than making your own copy. 
$30 \mathrm{~min}$. The melts of the VFQ type were pressed between two copper blocks, one of which was equipped with an internal cooling water system. The cooling period down to $200^{\circ} \mathrm{C}$ was estimated to be about 3-6 seconds. The glasses of the VSQ and SQ type were obtained by cooling the melts inside the furnace down to $200^{\circ} \mathrm{C}$ at a constant cooling rate of $7 \cdot 10^{-3}{ }^{\circ} \mathrm{C} / \mathrm{s}$ and $7.0 \cdot 10^{-2}{ }^{\circ} \mathrm{C} / \mathrm{s}$, respectively. All the samples were annealed for $2 \mathrm{~h}$ at $350^{\circ} \mathrm{C}$.

The compositions were checked by weighing the cooled melts: no significant variations of the weights were observed with respect to the expected values. Density measurements were carried out by the immersion method using chloroform as the immersion liquid. Quenching rates, compositions, densities and their errors are reported in Table 1, together with reference names used in the text.

The samples were grounded for the X-ray measurements with the exception of SQ glasses, which were measured as polished slabs. Details of $\mathrm{X}$-ray experiments and data treatments are given elsewhere [9-11].

Samples of a few tens of milligrams were broken from glass batches and analyzed with a Perkin-Elmer DSC7 calorimeter under flowing argon. The calorimeter cell was calibrated with pure In, $\mathrm{Zn}$ and $\mathrm{K}_{2} \mathrm{Cr}_{2} \mathrm{O}_{7}$ standard samples. $T_{\mathrm{g}}$ measurements were carried out using Platinum pans and with a heating rate $(q+)$ equal to the cooling rate $(q-)$ used during the glass preparation. According to the thorough demonstration of Moynihan et al. [12], this condition, different from the conventional one where the $q+$ is $10^{\circ} \mathrm{C} / \mathrm{min}$, leads to $T_{\mathrm{g}}$ values truly representative of the structural state of the system [12-13]. It is then obvious that the quenching rate during the preparation must be rigorously controlled. This is why the VFQ sample could not be examined. Therefore, a further sample, BONA2MQ, was prepared for the DSC measurements using a constant cooling rate of $1 \cdot 10^{-2}{ }^{\circ} \mathrm{C} / \mathrm{s}$ in order to have at least three good samples.

\section{Results and Discussion}

Structure and radial functions of the BONA2 and BONA3 samples are reported in Figs. $1 \mathrm{a}$ and $\mathrm{b}$ and Figs. 2a and b, respectively. All samples are completely amorphous; no traces of crystalline peaks are present, not even in the samples obtained by very slow cooling.
Table 1. Quenching rates, $q\left({ }^{\circ} \mathrm{C} / \mathrm{s}\right)$, compositions (wt $\left.\%\right)$ and densities, $d\left(\mathrm{~g} / \mathrm{cm}^{3}\right)$ of the six glasses investigated. Errors are given in parentheses. Reference names used in the text are given in column 1.

\begin{tabular}{lllll}
\hline Glass & $q$ & $\mathrm{~B}_{2} \mathrm{O}_{3}$ & $\mathrm{Na}_{2} \mathrm{O}$ & $d$ \\
\hline BONA2 VFQ & $2 \cdot 10^{2}$ & $69.2(1)$ & $30.8(1)$ & $2.336(1)$ \\
BONA2SQ & $7 \cdot 10^{-2}$ & $69.2(1)$ & $30.8(1)$ & $2.348(1)$ \\
BONA2 VSQ & $7 \cdot 10^{-3}$ & $69.2(1)$ & $30.8(1)$ & $2.370(1)$ \\
BONA3 VFQ & $2 \cdot 10^{2}$ & $77.1(1)$ & $22.9(1)$ & $2.234(1)$ \\
BONA3 SQ & $7 \cdot 10^{-2}$ & $77.1(1)$ & $22.9(1)$ & $2.241(1)$ \\
BONA3 VSQ & $7 \cdot 10^{-3}$ & $77.1(1)$ & $22.9(1)$ & $2.261(1)$ \\
\hline
\end{tabular}

Table 2. Structural parameters obtained for the first B-O coordination. Mean distances $r(\AA)$, root mean square deviations $\sigma(\AA)$ and mean coordination numbers $N$ are reported respectively in columns 2,3 , and 4 . Limit errors are given in parentheses.

\begin{tabular}{llll}
\hline Glass & $r$ & $\sigma$ & $N$ \\
\hline BONA2 VFQ & $1.436(5)$ & $0.08(1)$ & $3.0(2)$ \\
BONA2SQ & $1.426(5)$ & $0.07(1)$ & $3.0(2)$ \\
BONA2 VSQ & $1.435(5)$ & $0.09(1)$ & $3.0(2)$ \\
BONA3 VFQ & $1.416(5)$ & $0.07(1)$ & $3.0(2)$ \\
BONA3 SQ & $1.411(5)$ & $0.09(1)$ & $3.0(2)$ \\
BONA3 VSQ & $1.416(5)$ & $0.07(1)$ & $3.0(2)$ \\
\hline
\end{tabular}

The structure and radial functions are nearly identical for glasses with the same composition, both in the short and medium range order. Small differences, similar to the ones found in [8], are randomly distributed among the samples, and their magnitude is of the order of the experimental uncertainty.

In the radial distribution functions (RDF) two main peaks appear, centered at 1.40 and $2.40 \AA$ followed by shoulders at about $2.75,3.65,4.2$ and $4.6 \AA$. As already observed in previous studies on BONA samples [8-9], these functions are very similar to the RDF found for pure $\mathrm{B}_{2} \mathrm{O}_{3}[4,14-15]$, with differences arising from the cationic contributions and the network modifying action of the alkali oxide. A small spurious peak at $1.9 \AA$ is also present, due to termination errors in the RDF calculations. Spurious peaks at low $r$ are small and very similar, showing that systematic errors have been minimized.

To check the correctness of the results, the boron coordination was determined, as it is expected not to be affected by the thermal history and to depend on the composition only. The calculation was performed by simulating the Fourier backtransforms of the $\mathrm{B}-\mathrm{O}$ peak at $1.4 \AA$ in all systems. The values of the parameters from the best fits are given in Table 2. Errors were determined by comparing parameters obtained in sev- 


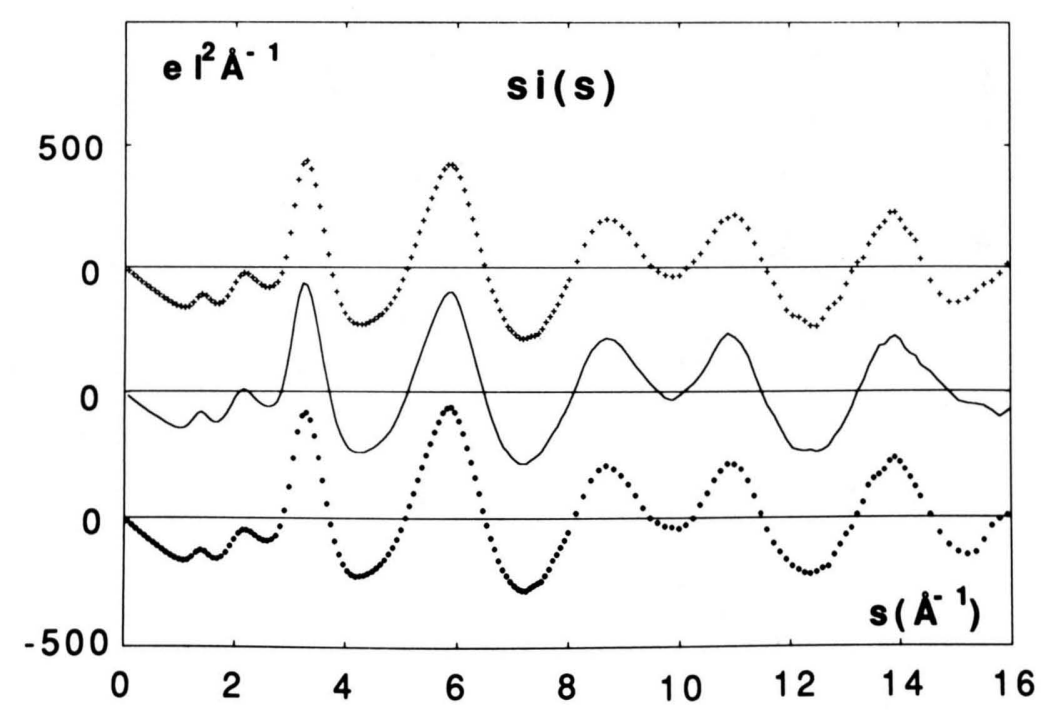

Fig. 1 a. Structure functions for BONA2 VFQ (dots), BONA2SQ (solid line) and BONA2 VSQ (crosses) glasses.

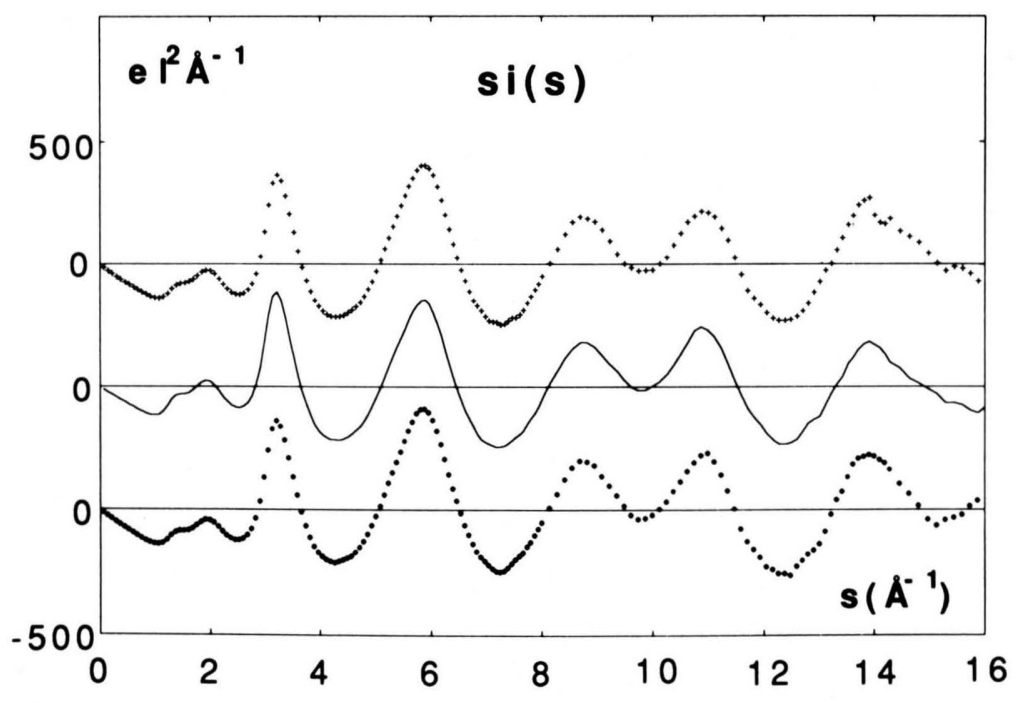

Fig. 1 b. Structure functions for BONA3VFQ (dots), BONA3SQ (solid line) and BONA 3 VSQ (crosses) glasses.

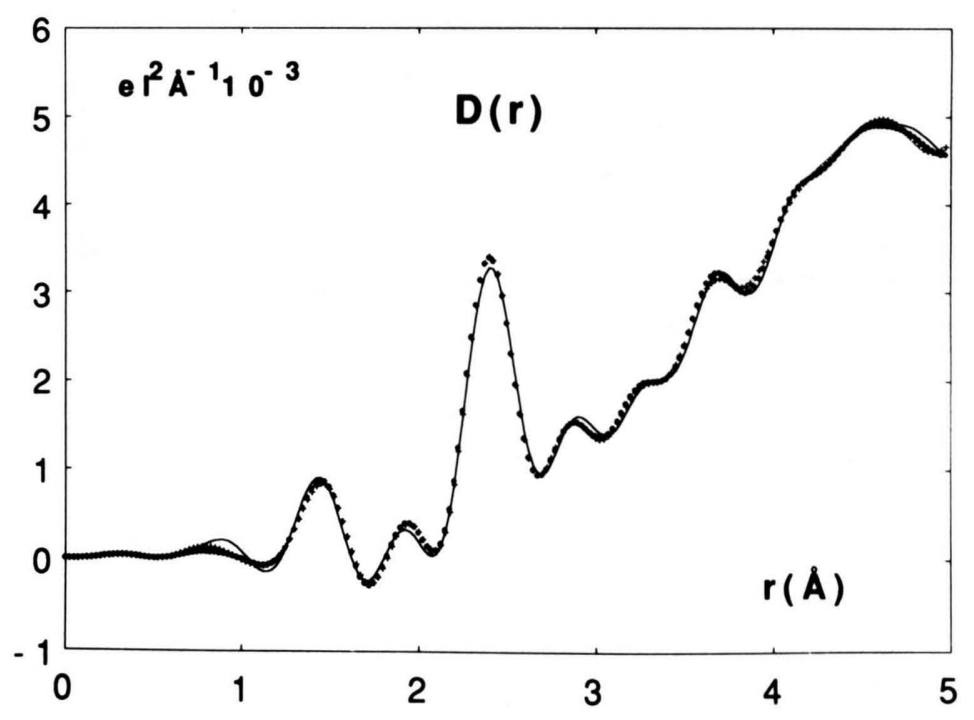

Fig. 2a. Radial distribution functions for BONA2VFQ (dots), BONA2SQ (solid line) and BONA2 VSQ (crosses) glasses.

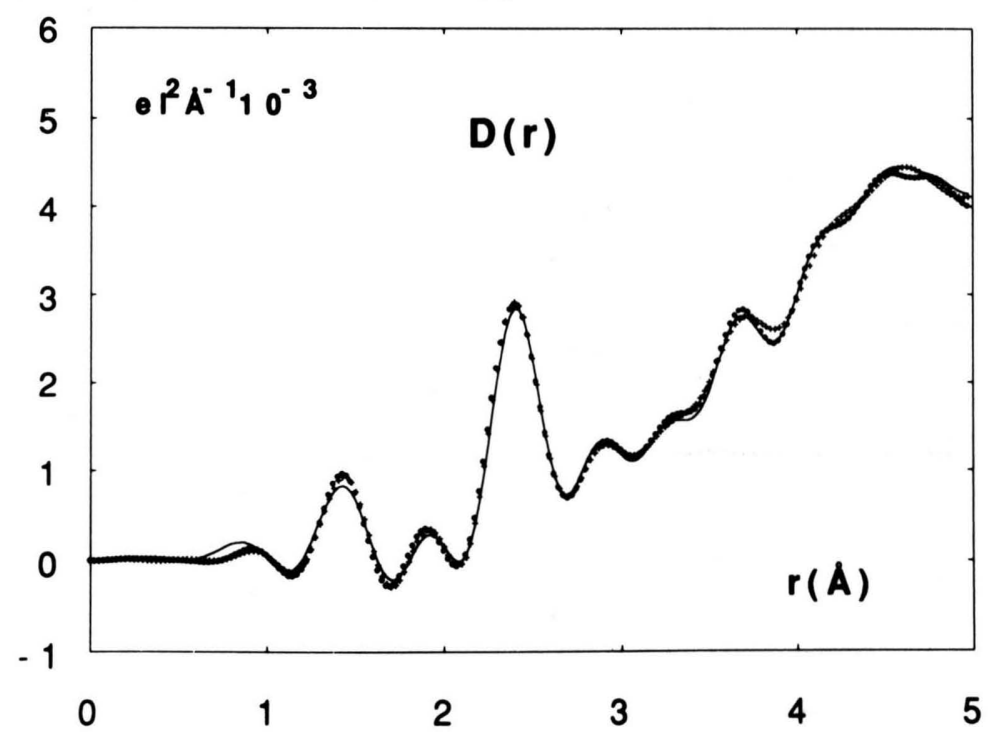

Fig. 2b. Radial distribution functions for BONA3 VFQ (dots), BONA3SQ (solid line) and BONA3 VSQ (crosses) glasses. 


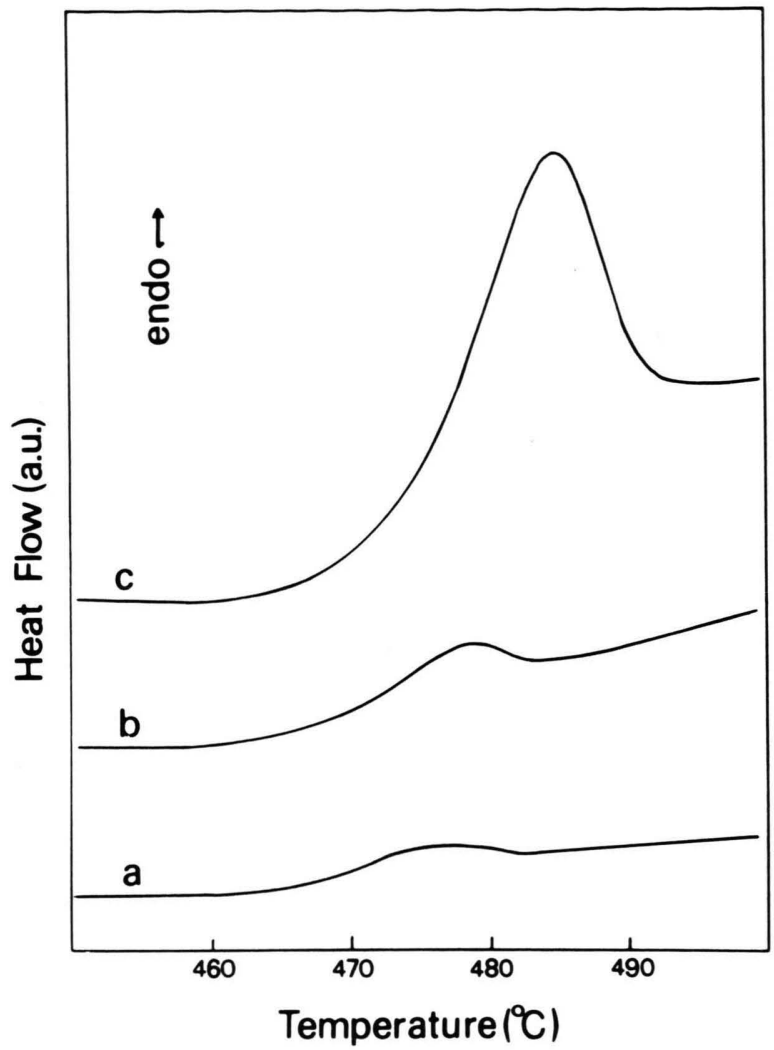

Fig. 3. DSC traces for BONA2 VSQ (a), BONA2MQ (b) and BONA2SQ (c) glasses.

eral calculations carried out using different ranges of experimental data or different refinement strategies.

As expected, the $\mathrm{B}-\mathrm{O}$ distance increases with sodium oxide content. In fact, in the explored composition range the alkaline oxide promotes the transformation of three-coordinated into tetra-coordinated boron atoms, and the $\mathrm{B}-\mathrm{O}$ distances in the $\mathrm{BO}_{4}$ units are $0.10-0.15 \AA$ longer than in the $\mathrm{BO}_{3}$ units. The coordination numbers, although rather reasonable, are a little lower than expected. As has been extensively discussed in [9], this is mainly due to the inadequacy of tabulated scattering factors, which are calculated for free spherical atoms, to represent the scattering power of light and covalently bonded atoms. No meaningful differences are noticed for different thermal treatment.

In search of possible effects of cooling rate on the structural properties of VSQ and VFQ glasses, a calculation of $\mathrm{Na}^{+}$coordination was performed on all the samples. In fact, contrary to the B-O first interac-
Table 3. Mean distances $r(\AA)$, root mean square deviations $\sigma(\AA)$ and frequency factors $N$ for the $\mathrm{Na}^{+}-\mathrm{O}$ interactions. Limit errors are given in parentheses.

\begin{tabular}{lllllll}
\hline Glass & $r_{1}$ & $\sigma_{1}$ & $N_{1}$ & $r_{2}$ & $\sigma_{2}$ & $N_{2}$ \\
\hline BONA2 VFQ & $2.370(5)$ & $0.09(1)$ & $2.5(2)$ & $2.71(1)$ & $0.11(1)$ & $1.5(1)$ \\
BONA2SQ & $2.394(5)$ & $0.11(1)$ & $2.5(3)$ & $2.74(1)$ & $0.15(1)$ & $1.7(1)$ \\
BONA2 2SQ & $2.378(5)$ & $0.09(1)$ & $2.5(3)$ & $2.72(1)$ & $0.13(1)$ & $1.6(1)$ \\
BONA3 VFQ & $2.401(5)$ & $0.13(1)$ & $3.0(3)$ & $2.77(1)$ & $0.17(1)$ & $1.3(1)$ \\
BONA 3SQ & $2.428(5)$ & $0.14(1)$ & $2.8(3)$ & $2.79(1)$ & $0.12(1)$ & $1.4(1)$ \\
BONA3 VSQ & $2.403(5)$ & $0.13(1)$ & $3.0(3)$ & $2.78(1)$ & $0.17(1)$ & $1.3(1)$ \\
\hline
\end{tabular}

\begin{tabular}{ll}
\hline Glass & $T_{\mathrm{g}}$ \\
\hline BONA2 2VSQ & $467(1)$ \\
BONA2 MQ & $468(1)$ \\
BONA2SQ & $472(1)$ \\
\hline
\end{tabular}

Table 4. Glass transition temperatures, $T_{\mathrm{g}}\left({ }^{\circ} \mathrm{C}\right)$ for BONA2 glasses. Standard errors are given in parentheses.

tion, the polyhedra of the modifier ion might be influenced by different preparation conditions.

To evaluate the coordination of the $\mathrm{Na}^{+}$ions it is necessary to extract from the total structure functions the contributions coming from the interactions $\mathrm{Na}^{+}$neighboring atoms. The difference method, in which the si(s) or the RDF of a suitably selected reference system is subtracted from the total function of the sample investigated, is often used to this end. Without discussing the details of the difference method, for which we refer to [9-11], we subtracted the RDF's of $\mathrm{Li}_{2} \mathrm{O} \cdot n \mathrm{~B}_{2} \mathrm{O}_{3}$ glasses (BOLI) from those of equimolar BONA samples in order to cancel the contributions of the borate network to the radial functions. Assuming that $\mathrm{Li}^{+}$ions give negligible contribution to the diffraction signal, the difference curves are representative of the local order around $\mathrm{Na}^{+}$ions.

The simulation of the $\mathrm{Na}^{+}-\mathrm{O}$ peak was performed using the same backtransform procedure as mentioned above. Very good fits were obtained with the parameters listed in Table 3. Two contributions were introduced with different distances, frequency factors and root mean square deviations; in fact the difference peak is not symmetric, showing that the $\mathrm{Na}^{+}$environment is not a regular polyhedron. The values obtained in the present study are consistent with [8-9]. Though some doubts on the absolute values could exist, we can observe that there is no discernible trend for glasses obtained by different cooling rates. This suggests that the $\mathrm{Na}^{+}$coordination polyhedron is not affected by the thermal history of the glass. 
Therefore, either by inspection of the entire structure or radial functions or by quantitative analysis of the main peaks of the radial curves, we can conclude that the examined glasses of the same composition show very small structural differences (if any). Let us now compare these results with those obtained by thermal analysis.

The DSC traces for BONA2SQ, MQ and VSQ samples, normalized to the unitary weight, are plotted in Figure 3.

The $T_{\mathrm{g}}$ values, obtained by the intersection of the tangent to the flex point and of the baseline extrapolation, are reported in Table 4 together with their limit errors. Several measurements were performed for each glass, with a reproducibility better than $1{ }^{\circ} \mathrm{C}$. The $T_{\mathrm{g}}$ values show a slight tendency to increase with the cooling rate used in the preparation or with the heating rate used in the DSC measurements [14]. This trend is consistent with the concept that FQ glasses have a more open and therefore easily alterable structure than SQ glasses, which are more compact. This fact can correlate with the densities reported in Table 1, which are slightly higher for VSQ glasses and progressively decrease going to VFQ glasses.

Then, some difference exists, though so slight as not to appear in the total diffractometric curves. The structural differences should appear in a distance range longer than that accessible to our analysis, or arise from differences in the coarse grain structure (dimensions of glassy domains, connections of these domains, phase separations and so on) which can be quite relevant for macroscopic properties.

The diffractometric results combined with densities and glass transition temperatures show that the extreme similarity of the different radial curves cannot be ascribed to poor sensitivity of the diffractometric technique in revealing variations in the medium range order in amorphous materials. As a matter of fact, the comparison of different radial distribution functions calculated for different structural models has demonstrated that they are significantly different in the medium range order, even when the first coordination is not affected [16]. Besides, it has been shown that, given the high reliability of diffraction data collected on modern diffractometers, structural models are unlikely to fit satisfactorily experimental data. These two points strongly suggest that medium range order in structurally different glasses leads to experimental radial distribution functions which are significantly different.
The observed high similarity of borate glasses prepared with different cooling rate is of great importance in evaluating the meaning of the structural models for vitreous substances. Since the evolution from undercooled melt to solid glass is not uniquely defined (that is, liquid to glass transition occurs at different temperatures if different cooling rates are adopted), every glass should have its own structure. While this is certainly true, the present result show that, once all the other parameters are fixed, glasses quenched differently are analogous structurally. The system, moving along the extremely complex potential energy hypersurface, tends towards a unique well defined state, that we may call ideal glass. This point has been introduced by researchers [17-18] who, on the base of the Kautzmann's idea [19] on the evolution of thermal capacity and excess entropy near the glass transition, conclude that the liquid-glass transition can be described as higher order thermodynamic transition kinetically hampered. Therefore, one can postulate the existence of an equilibrium vitreous state, the "ideal glass", whose attainment is frustrated by a question of time scale. Real glasses can then be thought of as perturbations of this ideal vitreous state.

It follows that structural models used to represent ideal vitreous states have the same meaning as perfect lattices representing crystalline substances: in both cases real structures can be considered as derived from these ideal situations by introducing defects of various kind. Quenching the glasses with different speeds would bring the final structure more or less close to the ideal one, which should be identical for all the samples.

\section{Conclusions}

The analysis of DSC results, together with the density values, suggest that configurational differences exist among glasses obtained with different cooling rates.

However, the RDF's of samples of the same composition are very similar both in the low and in the medium range order, and not even the coordination shell of the modifier ion changes going from VSQ to VFQ glasses. This result supports our previous study where, however, the difference between the higher and the lower cooling rates was within three orders of 
magnitude while in the present case it ranged from $2 \cdot 10^{2}{ }^{\circ} \mathrm{C} / \mathrm{s}$ to $7 \cdot 10^{-3}{ }^{\circ} \mathrm{C} / \mathrm{s}$.

On the basis of these considerations, it is possible to assume that the short and medium range order of these glasses is insensitive to the thermal history.

[1] E. I. Kamistos, A. P. Patsis, M. A. Karakassides, and G. D. Chryssikos, J. of Non-Cryst. Solids 126, 52 (1990).

[2] V. Gottardi, J. Non-Cryst. Solids 49, 461 (1982).

[3] W. Soppe, W. Ebens, and H. W. van den Hartog, J. Non-Cryst. Solids 105, 251 (1988).

[4] W. Soppe, C. van den Marel, W. F. van Gunsteren, and H. W. van den Hartog, J. Non-Cryst. Solids 103, 201 (1988).

[5] G. D. Chryssikos, E. I. Kamistos, and M. A. Karakassides, Phys. Chem. Glasses 31, 103 (1990).

[6] E. I. Kamistos and M. A. Karakassides, Phys. Chem. Glasses 30, 235 (1989).

[7] G. E. Walrafen, S. R. Samanta, and P. N. Krishnam, J. Chem. Phys. 72 (1), 113 (1980).

[8] M. P. Medda, A. Musinu, G. Paschina, and G. Piccaluga, J. Non-Cryst. Solids 150, 76 (1992).

[9] G. Paschina, G. Piccaluga, and M. Magini, J. Chem. Phys. 81, 6201 (1984).

[10] G. Licheri, A. Musinu, G. Paschina, G. Piccaluga, G. Pinna, and A. Magistris, J. Chem. Phys. 85, 500 (1986).

\section{Acknowledgements}

This work has been carried out within the Italian CNR Project "Materiali Speciali per Tecnologie Avanzate".

[11] M. Magini, G. Licheri, G. Paschina, G. Piccaluga, and G. Pinna, X-ray Diffraction Studies of Ions in Aqueous Solutions: Hydration and Complex Formation, CRC press, Boca Raton 1988.

[12] C. T. Moynihan, A. J. Easteal, J. Wilder, and J. Tucker, J. Phys. Chem. 78, 2673 (1974).

[13] G. W. Scherer, J. Non-Cryst. Solids 123, 75 (1990).

[14] R. L. Mozzi and B. E. Warren, J. Appl. Cryst. 3, 251 (1970).

[15] P. A. V. Johnson, A. C. Wright, and R. N. Sinclair, J. Non-Cryst. Solids 50, 281 (1982).

[16] A. C. Wright, G. Etherington, J. A. E. Desa, R. N. Sinclair, G. A. N. Connel, and J. C. Mikkelsen Jr., J. NonCryst. Solids 49, 63 (1982).

[17] C. A. Angell, J. Non-Cryst. Solids 131, 13 (1991).

[18] R. Parthaserothy, K. J. Rao, and C. N. R. Rao, Chem. Soc. Revs. 12 (4), 361 (1983).

[19] W. Kautzmann, Chem. Rev. 43, 219 (1948). 